\title{
Image Haze Removal Using Dark Channel Prior
}

\author{
ShaSha Liu, Xianghui Shen \\ Department of ECDAV Communication University of China, Beijing, China \\ dearsha@cuc.edu.cn shenxianghui@cuc.edu.cn
}

\begin{abstract}
In this paper,It mainly studied the method of image haze removal based on the dark channel prior and simulation realization about it. Experiment has proved that,the algorithm can according to the different concentration levels of fog to haze removal higher quality.This haze removal algorithm can keep the feeling of depth of the image, and reducing the real color of the image.

Index Terms - image enhancement haze removal dark channel prior
\end{abstract}

\section{Introduction}

In atomization images, the clarity of image details and color fidelity will decrease due to the two parts factors as atmospheric light scattering and transmittance. The quality of atmospheric light and transmittance,depends largely on the distance of the scene to the camera,therefore,atomization image degradation is associated with the distance of the scene to the camera.Haze removal image processing using dark channel prior,not only could according to the different concentration of the haze to processing images, but also could obtain a better quality of the depth map.This algorithm will have a better effect of haze removal for the atomization images such as traffic roads which have depth of features. In addition,this algorithm could improve the clarity of images, to achieve the reduction of color, as well as some of the enhanced processing for image details, and high quality haze-free images would be recovered.

\section{Traditional Image Haze Removal Algorithm}

Image haze removal methods are mainly divided into the two categories which are atomization image enhancement and atomization image restoration.The atomization image enhancement method is applicable to a wide range,but usually do not consider the physical causes of image degradation, the advantage is to be able to effectively improve the detail contrast of atomization images, that is for to stand out detail, but the information of the prominent part will cause some losses of varying degrees. These algorithms can be achieved through histogram equalization, homomorphic filtering, and the wavelet transform. Atomization image restoration methods, in order to obtain the estimated value of haze-free images without haze particles noise impact, first based on the physical processes of atomization image degradation,establish a mathematical model of image degradation, and use this model to do the reverse processing, so that the degradation of the atomization image is to be compensated. This method is highly targeted, generally there will be no loss of information, the key point is that the estimates of the parameters in the model. In
2009, He Kaiming put forward a dark channel prior method belongs to this [1] .

This paper mainly discussed the haze removal algorithm based on dark primary channel prior regular pattern,this algorithm has some significant advantages,such as removal haze high quality according to the different thickness of the haze,able to achieve the reduction of the color fidelity, and obtain a obvious depth maps. This algorithm is suitable for atomization images which have obvious depth features such as traffic roads images.

\section{Principle of Haze Removal Algorithm Based On The Dark Channel Prior}

The mathematical model of atomization images is:

$$
I(x)=t(x) J(x)+[1-t(x)] A
$$

Where $\mathrm{I}(\mathrm{x})$ represents the intensity values of atomization image, that is the actual filming atomization image used in the algorithm. $t(x)=e^{-\beta d(x)}$ is the transmittance, its physical meaning is that the portion of light which could not be scattered out, it through the propagation medium in the air and mapping to the camera. $\beta$ represents the atmospheric scattering coefficient, which reflects the different degree of atomization on the different locations in the images, that is for the thickness of the haze. As well as d represents the distance from the scene to the camera. Seen by the mathematical model, the atmospheric scattering coefficient $\beta$ and the depth coefficient $\mathrm{d}$ are the important factors to affect the transmittance,through the increase of the atmospheric scattering coefficient $\beta$ and the depth coefficient $d$, transmittance $\mathrm{t}(\mathrm{x})$ is exponentially attenuated. $\mathrm{A}$ is the atmospheric light components. $\mathrm{J}(\mathrm{x})$ represents the intensity of the light of the scene,that is the haze-free image which is waiting to be restored. The right side of term (1) is $t(x) J(x)$, it represents the attenuation portion,describes the attenuation portion of the image clarity which the light spreaded through the atmosphere medium and mapped to the camera;1-t(x) which on the right side of equation is the scattered portion after light through the atmosphere media.[1-t(x)]A is the atmosphere light component caused by scattering, showing the phenomenon that the color fidelity of image reduced.The substance of this atomization image enhancement algorithms through estimating the transmittance $t(x)$ and the atmospheric light components $A$ to restore the haze-free image $\mathrm{J}(\mathrm{x})$ from the atomization image $\mathrm{I}(\mathrm{x})$.

\footnotetext{
* This work is supported by Key Technologies Research and Development Program of China Grant \#2012BAH15B02-09.
} 
In the non-sky regions, there is always has at least one light intensity for a very low value in a local area, the minimum value is called dark channel.

$$
J_{\text {dark }}(x)=\min _{c \in(r, g, b)}\left\{\min _{y \in \Omega(x)}\left[J_{c}(y)\right]\right\}
$$

There are three color channels called $\mathrm{R}, \mathrm{G}, \mathrm{B}$ in the equation(2), $\mathrm{C}$ is one of the channels for the $\mathrm{R}, \mathrm{G}, \mathrm{B}, J_{c}$ is the intensity values of $\mathrm{J}$ in one of the three color channels, $\Omega_{x}$ is a local region that is take $\mathrm{x}$ as the center,y is a point in the local region.

In atomization images which are affected by the haze noise in the air,there always could be found some low intensity values of dark channel in the local region, but most of the haze is brightly.Because of the aggregation of white light in the atmosphere light, the dark channel value of haze will become very high, which is caused by the diffuse reflection component in the atmosphere,related to the intensity and transmittance. So dark channel could be directly used to evaluate the transmittance information of haze, could obtained high quality haze-free images and depth maps,as well as to determine the concentration of haze according to the gray degree of the dark channel point. This method could according the different distances of scene and the different concentration of haze on the different positions to deal with the scene detail informations of various parts targeted.

\section{Algorithm Implementation Steps For Image Haze Removal Based On The Dark Channel Prior}

Haze removal algorithm dark channel prior steps shown in Figure 1:

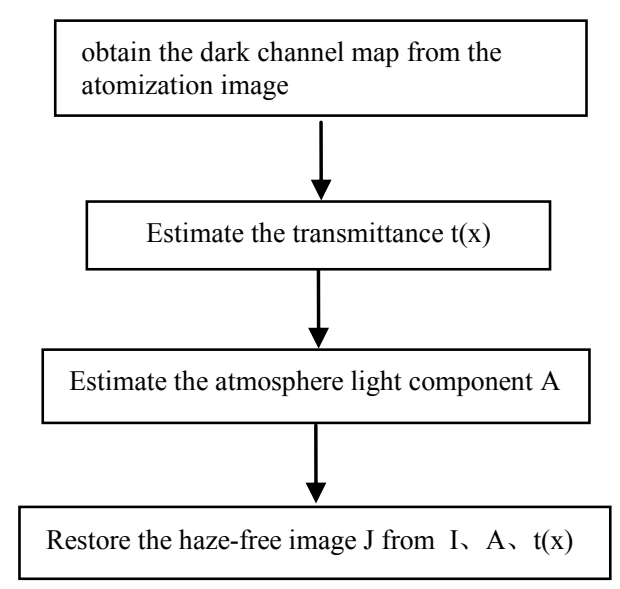

Fig. 1 Haze removal based on dark channel prior algorithm steps

The algorithm is divided into four steps. first of all,obtained the global dark channel diagram from the actual atomization image.Second, under the dark channel prior rule there will be found that there exsit at least one pixel dark channel value tends to zero in the haze-free images, and then socalculate the estimated value of the transmittance.Third step is to extracting the estimated value of the atmosphere light component A. Final step based on the estimated value of the atmospheric light component and the transmittance which is calculated,and recovery haze-free images from the atomization image haze removal enhancement processing.

\section{A. Dark Channel Values of Atomization Images}

Assuming atmospheric light A is known,and transmittance is unchangeably in a local region. Take the smallest operator on both sides of the equation (1) to the atomization image model and same divided by the atmospheric light A,drawn:

$$
\min _{y \in \Omega(x)}\left(\frac{I_{c}(y)}{A_{c}}\right)=t(x) \min _{y \in \Omega(x)}\left(\frac{J_{c}(y)}{A_{c}}\right)+(1-t(x))
$$

Then extract the minimum of three channels, there are:

$$
\min \left(\min _{y \in \Omega(x)}\left(\frac{I_{c}(y)}{A_{c}}\right)\right)=t(x) \min _{c}\left(\min _{y \in \Omega(x)}\left(\frac{J_{c}(y)}{A_{c}}\right)\right)+(1-t(x))
$$

Minimum in this single channel in the area, that is, the dark channel values of the local region. The important step in the above equation is that the regional block to the image, and then extracting the image dark channel value in the local region, the step parameters of regional block will directly affect the estimated results of transmittance $t(x)$. The smaller the area of the block, the transmittance estimated map will has more details, but will also add more detail error, and estimated value of atmospheric light will also occur error. If block area is too large, the error of the details will significantly reduce, but could lead to the details of the transmittance estimation map not prominent.Poor administrative levels feeling lead to the method can not get a high quality depth map. Consequently, in order to achieve a good balance between the detail presents of transmittance estimated and the error estimates, it is necessary to according to the specific circumstances of the actual images to adaptive block, this could enhance the effect of image haze removal.

\section{B. Transmittance}

According to the dark channel prior,we known that the dark channel value of the recovered haze-free image is close to zero. Therefore, Assume the value of $\min _{c}\left(\min _{y \in \Omega(x)}\left(\frac{J_{c}(y)}{A_{c}}\right)\right)$ in equation (4) is zero, derived the transmittance of the haze-free image:

$$
t(x)=1-\min _{c}\left(\min _{y \in \Omega(x)}\left(\frac{I_{c}(y)}{A_{c}}\right)\right)
$$

If thoroughly remove concentration of haze, the whole image will looked untruthfulness. The concentration of haze could always reflect to the information such as the distance of the objects to a certain extent.To completely remove the haze could also make the sense of depth decreases. Therefore, here you can set a parameter $\mathrm{k}(0<\mathrm{k}<1)$ to retain a part of the haze, make the scene form far covered with a small amount of haze which are supposed to be, so as to maintain the sense of depth. The transmittance after join the parameter $\mathrm{k}$ is: 


$$
t(x)=1-k \min _{c}\left(\min _{y \in \Omega(x)}\left(\frac{I_{c}(y)}{A_{c}}\right)\right)
$$

\section{Estimates of Atmospheric Light A}

Extract the $0.1 \%$ of the brightest pixels in the dark channel, the estimated value of the atmospheric light $\mathrm{A}$ is the extracted pixels from dark channel corresponding to the maximum luminance value in the atomization image I.

\section{Haze-free Image}

According to the transmittance $\mathrm{t}(\mathrm{x})$ and the atmospheric light A which are solved above,drawing the recovered hazefree image:

$$
J(x)=\frac{I(x)-A}{\max \left(t(x), t_{0}\right)}+A
$$

Because of $\mathrm{t}(\mathrm{x})$ is close to 0 , the restored haze-free image will has noise, we should set a threshold value $t_{0}$ to retain a certain amount of haze.

Dark channel prior regularity are drawn according to statistics.Regional pixels will meet the dark channel prior regularity if the image do not include the part of the sky. There could find a point in the region which has low dark channel values and is close to zero, and the recovery effect is ideality. For some images, such as lightness of the objects is very close to the sky, or larger than the brightness of the sky, the dark channel prior regularity will lapse.

\section{Achievement and Conclusions}

This paper based on the principle of dark channel prior algorithm, using VC6.0 development platform and the C language to processing the image haze removal.

The experimental results are shown in Figure 2.
Atomization image

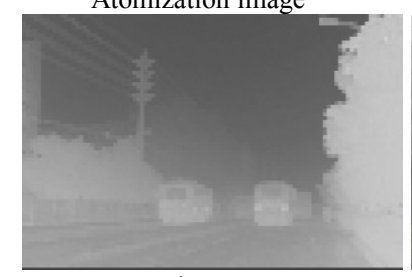

Transmittance map

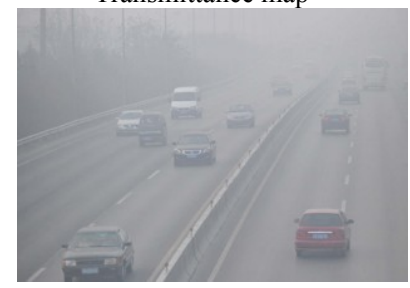

Atomization image

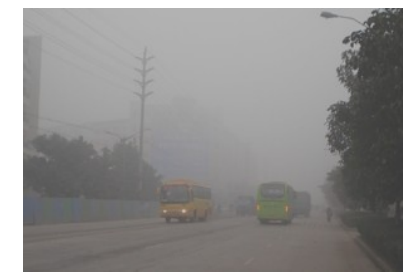

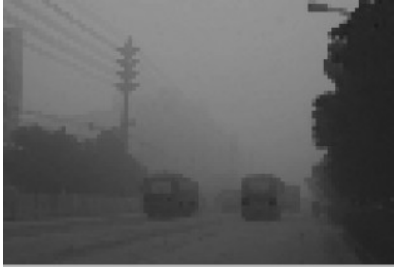

Global dark channel map

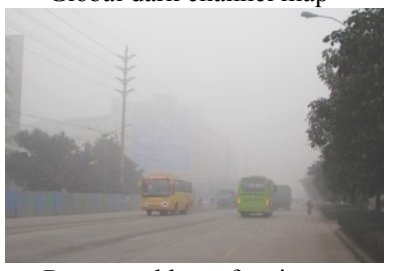

Recovered haze-free image

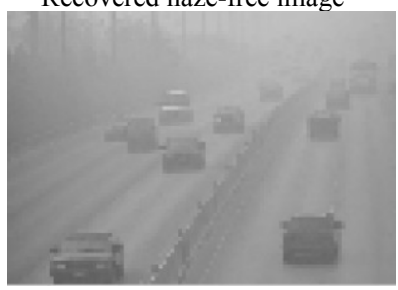

Global dark channel map

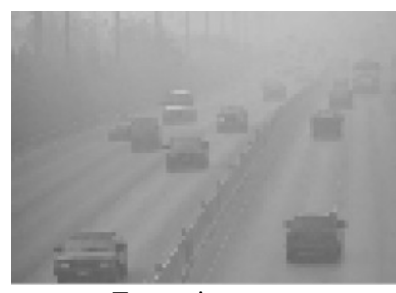

Transmittance map

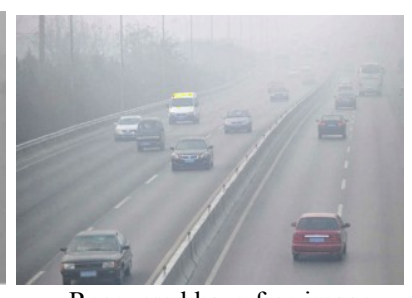

Recovered haze-free image
Fig. 2 Result of haze removal based on dark channel prior algorithm

This paper do lots of haze removal experiments to processing atomization images using the dark channel prior algorithm, the experimental results could achieve the theory of this algorithm. This algorithm could improve the blurred and unconspicuous details images due to haze in the images, to be able to restore the color well and maintain a sense of depth without distortion.

Bright region such as sky or partial white objects, is also bright in the case of haze-free, it is difficult to find the dark channel points which is close to 0 , so the assumption does not hold in this part of the region. To improve the effects of haze removal, it is need to expand the original algorithm, so that it can deal with the different scenes images.

After the image restoration, whether the transmittance of the haze-free image could continuously changed is related to the image block parameters. According to the study of $\mathrm{He}$ Kaiming [1], the introduction of a soft matting algorithm could improved the estimate of the transmittance, revise the recovered image blockiness, and could obtain better recovered images according to the contours of the object. But large amount of calculation for the soft matting algorithm cannot be used for real-time processing, it will be replaced,and there should looking for a new method such as the interpolation algorithm. Therefore, it is necessary to make an optimization algorithm for soft matting algorithm in order to better handle hardware to achieve the real-time video haze images removal processing.

\section{References}

[1] He Kaiming, Sun Jian, Tang Xiaoou. Single image haze removal using dark channel prior[C].IEEE Conference on Digital Object Identifier.2009:1956-1963.

[2] John P.Oakley,Brenda L.Satherley.Improving Image Quality in Poor Visibility Conditions Using a Physical Model for Contrast Degradation.IEEE Transactions on image Processing[C].1998,14(3):167179.

[3] KokKeong Tan,Oakley,J.P.Enhancement of Color Images in Poor Visibility Conditions.International Conference on Image Processing[C].2000,2:788-791.

[4] Srinivasa G. Narasimhan,Shree K. Nayar.Contrast Restortion of Weather Degraded Images.IEEE Transactions on Pattern Analysis and Machine Intelligence[C].2003,25(6): 713-724.

[5] JI Xiao-qiang,DAI Ming, SUN Li-na, LANG Xiaolong, WANG Hong. Research on the image haze-removal algorithm based on the prior darkchannel[J](in chinese). Journal of Optoelectronics laser,2011,06

[6] JIANG Jian-guo,HOU Tian-feng,QI Mei-bin.Improved algorithm on image haze removal using dark channel prior[J](in chinese). Journal of Circuits and Systems,2011,02 